\title{
Desferrioxamine decreases NAD redox potential of intact red blood cells: evidence for desferrioxamine as an inducer of oxidant stress in red blood cells Yutaka Niihara*1, Junli Ge ${ }^{1}$, Oded Shalev², Huiyuan $\mathrm{Wu}^{1}{ }^{1}$, Amy Tu${ }^{1}$ and Kouichi R Tanaka
}

Address: ${ }^{1}$ Department of Medicine, Harbor-UCLA Medical Center, UCLA School of Medicine, Torrance, California, USA and ${ }^{2}$ Department of Medicine, Hadassah-Mt. Scopus, Jerusalem, Israel

E-mail: Yutaka Niihara* - ysniihara@msn.com; Junli Ge - ysniihara@msn.com; Oded Shalev - odeds@cc.huji.ac.il; Huiyuan Wu - ysniihara@msn.com; Amy Tu - ysniihara@msn.com; Kouichi RTanaka - kthucla@aol.com

${ }^{*}$ Corresponding author

Published: 24 October 2002

BMC Clinical Pharmacology 2002, 2:8

This article is available from: http://www.biomedcentral.com/1472-6904/2/8

(C) 2002 Niihara et al; licensee BioMed Central Ltd. This article is published in Open Access: verbatim copying and redistribution of this article are permitted in all media for any purpose, provided this notice is preserved along with the article's original URL.
Received: 15 April 2002

Accepted: 24 October 2002

Keywords: red blood cells, desferrioxamine, NAD redox potential, oxidant stress, iron

\begin{abstract}
Background: Desferrioxamine (DFO) is an important iron chelating agent. It has also been thought of as an agent with anti-oxidant potential as it chelates ferric iron in various parts of the body. However, there is evidence suggesting that it may paradoxically affect red blood cells (RBC) by inducing intracellular oxidant stress. To further understand the mechanism of DFO's interaction with RBC, we conducted a study to determine the effect of DFO upon RBC's redox status.
\end{abstract}

Methods: We examined NAD redox potential in intact $R B C(N=5)$ incubated with DFO. RBC were incubated with $6 \mathrm{mM}$ DFO for 2 hours.

Results: Significant decreases in NAD redox potential were observed after incubation of RBC with $6 \mathrm{mM}$ DFO. The mean decrease was $10.01 \pm 1.98 \%(\mathrm{p}<0.0004)$.

Conclusions: The data confirm the oxidant effect of DFO on RBC.

\section{Introduction}

Desferrioxamine (DFO) currently is the only iron chelator that is available for clinical use in the United States. It is a potent chelator that was originally obtained from an ironbearing metabolite of Actinomyceles (Streptomyces pilosus) with specificity for ferric iron $\left(\mathrm{Fe}^{3+}\right)[1,2]$. Its stability constant of $10^{31}$ for ferric iron contrasts significantly with those for other ions such as zinc $\left(10^{11}\right)$, calcium $\left(10^{2}\right)$ and magnesium $\left(10^{4}\right)[2]$. Studies have demonstrated the ability of DFO to remove iron from various iron pools including ferritin and hemosiderin in iron-overloaded pa- tients. DFO also competes with unsaturated transferrin and removes non-transferrin bound iron [3].

DFO has also become known as an anti-oxidant. It is well recognized that iron-mediated oxidant stress leads to lipid peroxidation of the cell membrane and other cellular or tissue damage[4,5]. Iron excess can occur at the tissue or cell level with or without generalized iron overload as seen with redox cycling compounds that can liberate iron from ferritin [5]. Therefore, lipid peroxidation can occur without generalized iron overload [6]. It is thought that 
Table I: Net change of NADH/NAD ratio (\%) in blood samples incubated 2 hours with DFO

\begin{tabular}{lccc}
\hline & \multicolumn{2}{c}{ DFO concentration } & Net Change \\
NADH/NAD & $0 \mathrm{mM}$ & $6 \mathrm{mM}$ & $0 \mathrm{mM}-6 \mathrm{mM}$ \\
Ratio (\%) & $75.89 \pm 14.01$ & $65.88 \pm 12.19$ & $10.01 \pm 1.98 \mathrm{p}<0.0004$ \\
\hline
\end{tabular}

$n=5$ DFO, Desferrioxamine

elimination of free iron by a chelating agent such as DFO is vital in preserving the integrity of the cell membrane with localized or generalized free iron excess.

The observation that DFO protects tissues from oxidant stress has become an important and well-regarded factor in understanding its molecular qualities. However, there is another aspect of DFO that is not as well recognized. As one looks closer at the nature of DFO, it becomes apparent that it also acts as a prooxidant molecule. Studies of the redox mechanism have demonstrated that DFO increases iron dependent radical formation [7]. There are also data supporting DFO as being the cause of damage to tissues [2,7-12], including a report of DFO aggravating inflammation and exacerbating the toxicity of certain xenobiotics [2]. Therefore, on one hand, DFO may be an effective scavenger of potentially oxidant substances in certain parts of the body, but on the other hand, it may also act as a prooxidant in other areas such as the intracellular environment of red blood cells.

Previously, we have published the data demonstrating that DFO conjugated with starch leads to decrease in NAD redox potential[13]. As conjugation with starch makes DFO practically impossible to penetrate into RBC, it supported a notion that the oxidant stress was exerted from outside of RBC. In the study presented here, our data further demonstrate the prooxidant nature of DFO. This current data confirms previous studies and shows that DFO by itself without conjugation with starch does exert oxidant stress.

\section{Methods \\ Materials}

Spectrophotometric grade ethanol was purchased from Aldrich Chemical Co. (Milwaukee, WI). Desferoxamine Mesylate (Sigma, catalogue number D-9533), alcohol dehydrogenase, (Sigma, catalogue number A-3263) and all other reagents were purchased from Sigma Chemical Co. (St. Louis, MO).

\section{Study subjects and blood samples}

After proper consent, five normal healthy adults volunteered to participate in the study. Blood samples were obtained by routine venipuncture using heparin-coated tubes (15 $\mathrm{u}$ heparin/ml whole blood) to prevent coagulation.

\section{DFO incubation and pyridine nucleotide extraction}

Freshly drawn heparinized whole blood (425 $\mu \mathrm{l})$ was incubated in a $37^{\circ} \mathrm{C}$ water bath with DFO in saline buffer. The final volume of the incubation sample was $500 \mu \mathrm{l}$ with final concentrations of DFO at $0 \mathrm{mM}$ and $6 \mathrm{mM}$. Samples were collected at 2 hours of incubation and extracts were prepared immediately. Twenty $\mu$ l of incubation sample were mixed with $1,980 \mu \mathrm{l}$ of a solution containing $10 \mathrm{mM}$ nicotinamide, $20 \mathrm{mM}$ NaHCO3, and $100 \mathrm{mM} \mathrm{Na}_{2} \mathrm{CO}_{3}$ at $0^{\circ} \mathrm{C}$. The mixture was frozen in a dry ice-acetone bath for 20 minutes, then thawed quickly in a room temperature water bath, and promptly chilled to $0^{\circ} \mathrm{C}$. To destroy the oxidized form of NAD, $700 \mu \mathrm{l}$ of this mixture was incubated at $60^{\circ} \mathrm{C}$ for 30 minutes. The mixture was then chilled to $0^{\circ} \mathrm{C}$ for 1 minute. Both the heattreated extract that contained NADH and the unheated extract that contained both the reduced and oxidized forms of NAD were immediately analyzed using spectrophotometric cycling assays [14].

\section{NADH and total NAD assays}

NAD was assayed using spectrophotometric enzymatic cycling assays that measure both the oxidized and reduced forms of the nucleotide as previously described[14,15].

\section{Statistical analysis}

Paired t-test was used to evaluate the differences of NAD/ $\mathrm{NADH}$ ratio values with and without DFO incubation.

\section{Results}

We evaluated blood samples from five normal volunteers. There were consistent decreases in NAD redox potential with $6 \mathrm{mM}$ DFO-starch at 2-hour incubations. Mean decrease was $10.01 \pm 1.98 \%(\mathrm{p}<0.0004)$ (Table 1$)$.

Figure. 1 illustrates the change in NAD redox potential of each sample with DFO incubation.

\section{Discussion}

In this study, we demonstrated a decrease in NAD redox potential in normal RBC by incubation with DFO, although we must note that the in vitiro concentration DFO 


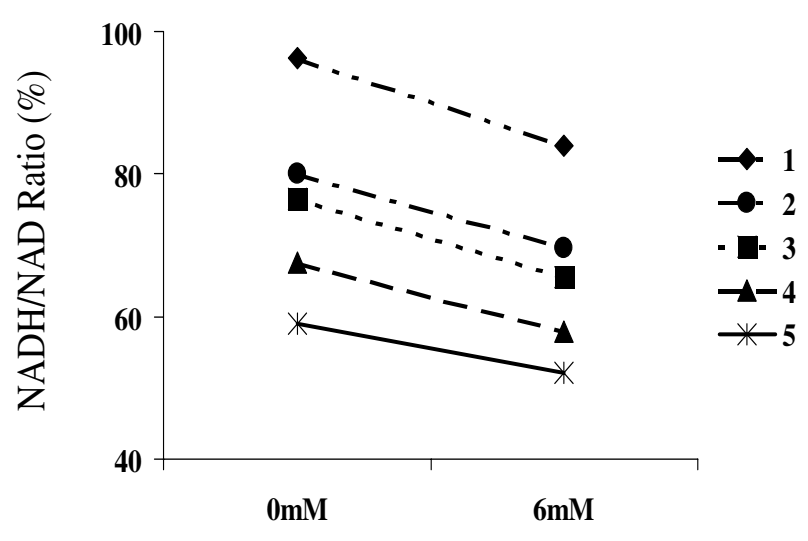

DFO Concentration

\section{Figure I}

NADH/NAD ratio (\%) from blood samples incubated 2 hours with DFO.

used for the assay here is significantly higher than what is expected with usual dosage used in clinical practice. Nonetheless, as the NAD system comprises the major factor in intracellular antioxidant activity within the RBC, this observation suggests the prooxidant effect of DFO upon the cytosol of RBC[16]. In regard to the mechanism of the prooxidant effect, because DFO is poorly permeable to RBC membrane [3], the action of DFO may primarily originate extracellularly to cause changes in the intracellular NAD redox potential. In fact, this concept of extracellular oxidant stress was confirmed in previously published data using DFO conjugated with starch to increase the molecular weight [13]. With conjugation with starch, it becomes impossible for the DFO to enter into RBC. Therefore the oxidant stress, if present, must come from outside of RBC yet it affects NAD which is inside the RBC. The data presented here is to make certain that DFO by itself without conjugation with starch leads to a decrease in NAD redox potential.

There is a report of potential prooxidant effect of iron saturated DFO solution [2]. Thus, one may consider the possibility of iron saturated DFO rather than DFO alone to be the source of oxidant stress. It is possible that there is a small fraction of iron bound DFO in the assay that may exert oxidant stress. However, in our experiment, there is no iron added to the solution. Therefore, although it is speculative, with the amount of DFO used in the assay, it is practically impossible for DFO to have scavenged membrane iron and become saturated. In addition, because RBC samples are from normal volunteers without any known disease processes, it is very unlikely that there is a significant amount of excess iron.
Several mechanisms may be considered to explain the effect of DFO on cytosol NAD redox potential. A potential mechanism is that extracellular DFO is causing an increase in ferric iron in RBC. It has been suggested for decades that the reaction between ferrous ion and DFO involves oxidation of iron to the ferric state prior to complexation [1]. Previous reports have demonstrated that under oxygen-free conditions, there is no spectrophotometric evidence (200-760 mu) for the formation of a complex between ferrous sulfate and DFO. When the mixture of iron and DFO was subsequently exposed to air or oxygen, it led to the formation of a ferric complex [1]. These experimental results lend support to the notion that DFO is likely to be involved in the conversion of ferrous into ferric iron before the formation of complexes.

As these data imply that DFO induces conversion of ferrous to ferric iron prior to the formation of DFO-iron complex [1], one may hypothesize that DFO may exert its oxidant effect on iron without coming in contact with the iron. Furthermore, DFO is perhaps able to oxidize iron that is on the other side of the RBC membrane. As a consequence, DFO removes membrane ferric iron, yet at the same time, it may oxidize cytosol ferrous iron into ferric iron. In this manner, DFO may behave as an antioxidant as well as a prooxidant. A way to test this hypothesis may be to study any change in methemoglobin level and cytosol iron state before and after incubation with DFO.

There are reports that lipid peroxidation can be stimulated by both free and complexed iron $[17,18]$. It is well documented that iron has an important role in oxidation and inflammatory process. However, at the same time the evidence shows that DFO with or without iron also appears to contribute in the oxidation process [10,19-21]. Also, there are other clinical data demonstrating that iron-free DFO, rather than iron complexed DFO, can be the culprit for toxicity $[7,19]$. Borg and Shariah have suggested that DFO itself would exert prooxidant action by producing highly reactive and potentially cytotoxic hydroxy radicals [19]. Subsequently, electron spin resonance (ESR) spectroscopy studies have detected and identified a DFO nitroxide free radical generated by the reactions with UV radiation [20], superoxide [10,21], and hydroxy radicals. According to studies by Mordente et al., in air-saturated solution, DFO appears to mediate one electron transfer from ascorbate to oxygen, with generation of superoxide anions leading to production of desferal nitroxide radicals or the Fenton reaction: $\mathrm{Fe}^{2+}+\mathrm{H}_{2} \mathrm{O}_{2} \rightarrow \mathrm{OH}+\mathrm{Fe}^{3+}+\mathrm{OH}^{7}$. The data presented in this paper concur with those reports suggesting prooxidant activities of DFO. This does not necessarily contradict the conventional wisdom of DFO as an anti-oxidant in various aspects of biological systems. However, it does provide an additional perspective that allows us to better understand the potential effects and 
toxicity of the compound, which is essential for the appropriate application of this product.

DFO therapy has become a vital part in the management of patients who require chronic transfusion therapy. Unless a better agent becomes available, DFO will continue to be "the chelator" for these patients. Fortunately, DFO has been the most effective and relatively safe iron chelator for patients with transfusional iron overload. However, as this medication is usually used on a chronic basis for the patients, we need to continue to gather data on the long term effects which may be attributable to DFO. In addition, since large numbers of patients who require iron chelation therapy are those with RBC that are more susceptible to oxidant stress (i.e., sickle cell disease, thalassemia), a clear understanding of the redox effect of DFO cannot be over emphasized.

\section{Conclusions}

We observed a decrease in NAD redox potential of normal RBC when incubated with DFO. This suggests a prooxidant activity of DFO upon the intracellular environment of RBC. Prooxidant activity of DFO is less well known and reported compared to its antioxidant activity. However, the evidence for prooxidant activity of DFO has been firmly demonstrated previously, both with iron saturated and unsaturated DFO. The factors affecting DFO to exert prooxidant activity or antioxidant activities appear to vary. Therefore, the two-sided observation of this single molecule does not necessarily indicate a contradiction of one from the other. In fact, they may complement each other. Clinically, because DFO is used in large numbers of patients, we need further understanding of the compound. This is particularly true in patients with oxidant susceptible RBC such as in sickle cell anemia and thalassemia.

\section{References}

I. Goodwin JF, Whitten CF: Chelation of ferrous sulphate solutions by desferrioxamine B. Nature 1965, 205:28I (Abstr)

2. Blake DR, Hall ND, Bacon PA, Dieppe PA, Halliwell B, Gutteridge JM: Effect of a specific iron chelating agent on animal models of inflammation. Ann Rheum Dis 1983, 42:89-93

3. Hamilton KO, Stallibrass L, Hassan I, Jin Y, Halleux C, Mackay M: The transport of two iron chelators, desferrioxamine $B$ and LI, across Caco-2 monolayers. Br J Haematol 1994, 86:85 I-7

4. Minotti G, Aust SD: The role of iron in the initiation of lipid peroxidation. Chem Phys Lipids 1987, 44:191-208

5. Rachidi S, Coudray C, Baret P, Gelon G, Pierre JL, Favier A: Inhibition of lipid peroxidation by a new family of iron chelators. Comparison with desferrioxamine. Biol Trace Elem Res 1994, 41:77-87

6. Winterbourn CC, Vile GF, Monteiro HP: Ferritin, lipid peroxidation and redox-cycling xenobiotics. Free Radic Res Commun I99I, I2-I3(Pt I): I07-14

7. Klebanoff SJ, Waltersdorph AM, Michel BR, Rosen H: Oxygenbased free radical generation by ferrous ions and deferoxamine. J Biol Chem 1989, 264:19765-7I

8. Mordente A, Meucci E, Miggiano GA, Martorana GE: Prooxidant action of desferrioxamine: enhancement of alkaline phosphatase inactivation by interaction with ascorbate system. Arch Biochem Biophys 1990, 277:234-40
9. Blake DR, Winyard P, Lunec J, Williams A, Good PA, Crewes SJ, Gutteridge JM, Rowley D, Halliwell B, Cornish A, et al: Cerebral and ocular toxicity induced by desferrioxamine. $Q J$ Med 1985 , 56:345-55

10. Davies MJ, Donkor R, Dunster CA, Gee CA, Jonas S, Willson RL: Desferrioxamine (Desferal) and superoxide free radicals. Formation of an enzyme-damaging nitroxide. Biochem J 1987, 246:725-9

II. Yoshino S, Blake DR, Bacon PA: The effect of desferrioxamine on antigen-induced inflammation in the rat air pouch. $J$ Pharm Pharmacol 1984, 36:543-5

12. Bergamini S, Rota C, Staffieri M, Tomasi A, lannone A: Prooxidant activity of ferrioxamine in isolated rat hepatocytes and linoleic acid micelles. Chem Res Toxicol 1999, I 2:365-70

13. Niihara Y, Shalev O, Hebbel RP, Wu H, Tu A, Akiyama DS, Tanaka KR: Desferrioxamine (DFO) conjugated with starch decreases NAD redox potential of intact red blood cells (RBC): evidence for DFO as an extracellular inducer of oxidant stress in RBC. Am J Hematol 2000, 65:28I-4

14. Zerez CR, Lee SJ, Tanaka KR: Spectrophotometric determination of oxidized and reduced pyridine nucleotides in erythrocytes using a single extraction procedure. Anal Biochem 1987, I 64:367-73

15. Niihara Y, Zerez CR, Akiyama DS, Tanaka KR: Oral L-glutamine therapy for sickle cell anemia: I. Subjective clinical improvement and favorable change in red cell NAD redox potential. Am J Hematol 1998, 58: I 17-21

16. Jaffe ER, Hultquist DE: Metabolic and Molecular Basis of Inherited Disease. Cytochrome b5 reductase deficiency and enzymopenic hereditary methemoglobinemia. New York, McGrawHill (Edited by: CR Scriver, AL Beaudet, WS Sly, D Valle) I995, 3399-34 I 5

17. Gutteridge JM, Paterson SK, Segal AW, Halliwell B: Inhibition of lipid peroxidation by the iron-binding protein lactoferrin. Biochem J 1981, 199:259-6I

18. Wills ED: Mechanisms of lipid peroxide formation in animal tissues. Biochem J 1966, 99:667-76

19. Hinojosa O, Jacks TJ, Calamari TA, Thibodeaux DP: Electron spin resonance study of the relationship of UV irradiation to nitroxide radical formation in desferrioxamine (Desferal) and related hydroxamate and hydroxylamine derivatives. Photochem Photobiol 1989, 49:1-5

20. Borg DC, Schaich KM: Prooxidant action of desferrioxamine: Fenton-like production of hydroxyl radicals by reduced ferrioxamine. J Free Radic Biol Med 1986, 2:237-43

21. Morehouse KM, Flitter WD, Mason RP: The enzymatic oxidation of Desferal to a nitroxide free radical. FEBS Lett 1987, 222:24650

\section{Pre-publication history}

The pre-publication history for this paper can be accessed here:

http://www.biomedcentral.com/1472-6904/2/8/prepub

\footnotetext{
\begin{tabular}{|} 
Publish with BioMed Central and every \\
scientist can read your work free of charge \\
"BioMedcentral will be the most significant development for \\
disseminating the results of biomedical research in our lifetime." \\
Paul Nurse, Director-General, Imperial Cancer Research Fund \\
Publish with BMC and your research papers will be: \\
• available free of charge to the entire biomedical community \\
• peer reviewed and published immediately upon acceptance \\
- cited in PubMed and archived on PubMed Central \\
• yours - you keep the copyright \\
Submit your manuscript here: \\
http://www.biomedcentral.com/manuscript/
\end{tabular}
} 